\title{
EDITORIAL
}

\section{Contact Tracing: A Forgotten Tool?}

\author{
Prof. Maryam Riaz Tarar ${ }^{1}$, Dr. Saadia Shahzad ${ }^{2}$ \\ Professor, Department of Pathology, Shalamar Medical \& Dental College, Lahore ${ }^{1}$ \\ Associate Professor, Department of Community Medicine, Shalamar Medical \& Dental College, Lahore ${ }^{2}$
}

Doi: doi.org/10.53685/jshmdc.v2i1.18

Contact tracing is an activity in public health domain, which is undertaken by experienced staff with an individual who has a communicable disease, identified by diagnostic testing. The process involves detailed interviewing of the infected person or the "Index case" who has been diagnosed with a notifiable disease, with a view to identify those people (contacts) who have been in contact with him/her. The objective is to interrupt the spread or transmission of the causative agent to others who are in close contact with the index case and are vulnerable by not being immune. Therefore, this acts as an important tool in controlling the spread of infection in a particular household or a community at large. Public health experts have relied on contact tracing to reduce the spread of infections like tuberculosis, blood borne viral infections including HIV (human immunodeficiency virus), sexually transmitted infections and measles ${ }^{1}$.

In a different dimension, in the present Corona virus pandemic that started last year in March 2020, contact tracing data has been used extensively by public health organizations in countries like China and UK to determine secondary attack rates in their populations ${ }^{2-3}$. This data has not only formed the basis of international meta-analysis publications but has also resulted in appropriate strategic public health responses. However, healthcare systems in developing countries that are underfunded and ill-equipped to respond to natural disasters or pandemics of this kind have not been able to make effective use of this tool of infectious disease control. Contact tracing in healthcare sector can be of household members or of professional colleagues at the workplace; both will enable to control $\mathrm{R}_{0}{ }^{4}$.

Our private healthcare organization has more than two thousand employees in different cadres (medical, nursing, allied \& support staff). Some of the staff started getting infected with Corona virus during the first wave of infections that started gaining momentum around June 2020, after the lockdown period (March-April 2020) ended in Pakistan. The need was felt in the organization to revisit the hospital policies regarding provision of support services for those healthcare workers (HCWs) who were getting infected and may require additional time being off sick, to recover. This included major subsidy by the organization for conducting diagnostic testing for all HCWs. It was also realized that in addition to those who were infected with Corona virus, there were other HCWs who were apprehensive about having possible exposure to these index cases at work and needed immediate guidance. This prompted the Infection Control Team (ICT) to initiate a process of contact tracing by gathering personal and exposure-specific information from both the laboratory confirmed index case HCW / source patient and all those employees who had been significantly exposed to this index case at workplace, as contacts.

Contact Tracing Tool: A contact tracing tool was developed by ICT on lines of conventional contact tracing for infectious diseases that included: 1) Socio demographics: name of the exposed HCW/contact (employee code); Exposure time \& date (including details about index HCW/source patient); Interview time \& date (interviewer). 2) Exposure-specific questions: wearing mask at the time of exposure (index case/ source 
patient); distance between the exposed HCW/ contact, from the source; duration of exposure of the contact; type of personal protective equipment worn by the exposed HCW/ contact at the time of exposure; hand hygiene status of the exposed $\mathrm{HCW} /$ contact at the time of exposure; exposed HCW/ contact have touched their face after exposure; type of procedures performed by the HCW/ contact in past 07 days; history of exposure with another lab-confirmed Corona virus case; if exposed HCW/ contact been tested for corona virus antibodies.

Once the Corona virus PCR (polymerase chain reaction) positive result of a HCW was shared with ICT by the Pathology Laboratory, the HCW was contacted by Infection Control Nurse (ICN) for an interview. First-hand information was obtained in detail from all index cases / source patients, through phone calls. Information about all those HCWs who may have been inadvertently exposed to the index case during the previous 1-2 weeks period was obtained from the index HCW. The named contacts were then approached by ICN to gather information from them and to alert them to the possibility of infection. Once the contact tracing forms were completed, the information was individually scrutinized by Director Infection Control Unit (MRT) to determine the extent and type of exposure of the contact with the index case. Depending on this, diagnostic testing was suggested to those HCWs who had prolonged close contact with index case and were considered as high risk exposure. The contact tracing was confined to work contacts only though information was provided to the exposed contacts regarding isolation practices to be followed at home. It would have been ideal to have extended this contact tracing exercise for home contacts but it was not possible to do so due to staff shortage. From the month of April 2020 onwards to 31 May 2021, a total of 210 HCWs contracted laboratory confirmed Corona virus infection, in our organization. For each positive index case, contact tracing was performed for 230 exposed HCWs, on average. Personalized advice was offered to all employees initially by members of ICT with further support by referral to Department of Pulmonology, for those contacts who did experience Corona virus infection. We hope to have in-depth analysis of the contact tracing data of our organization for a separate publication.

To conclude, this traditional contact tracing tool has been used effectively for controlling spread of communicable diseases in the workforce in our healthcare facility. It formed the basis of a strong support system for all those employees who contracted Corona virus infection and needed urgent advice about isolation/ quarantine or about the preventive measures at the household level for their families. A dashboard system for reporting Corona virus infections in our employees was also developed successfully by Human Resource Department at our institution; that greatly helped in keeping log of the cases at the organization level; guiding towards the work policy in case of HCW diagnosed with COVID; registration of the organization employees for vaccination; and keeping a record of the completed vaccination status of the employees.

\section{References:}

1. CDC (Centers for Disease Control and Prevention). Interim Guidance on Developing a COVID-19 Case Investigation \& Contact Tracing Plan: Overview. 2021. Available from: https://www.cdc.gov/coronavirus/2019-ncov/php/contact-

tracing/contact-tracing-plan/overview.html (accessed 23.05.2021)

2. PHE (Public Health England). SARS-CoV2 susceptibility and transmission risk in children: an overview of current evidence from Public Health England's surveillance work. 2020. Available at:

https://assets.publishing.service.gov.uk/government/uploads/system/uploads/attachment_data/file/916891/phe-susceptibilitytransmission-children-s0717-sage-53-200819.pdf (accessed 23.05.2021) 
3. PHE (Public Health England). Investigation of Novel SARS-CoV-2 variant- a Technical Briefing. Available at: https://assets.publishing.service.gov.uk/government/uploads/system/uploads/attachment_data/file/959360/Variant_of_Conce rn_VOC_202012_01_Technical_Briefing_3.pdf (accessed 23.05.2021)

4. Althoff KN, Coburn SB, Nash D. contact tracing: Essential to the Public Health Response and Our Understanding of the Epidemiology of Coronavirus Disease. Clin Infec Dis. 2019; 71(8): 1960-61.

\section{Corresponding Author:}

Dr. Saadia Shahzad

Associate Professor

Department of Community Medicine

Shalamar Medical \& Dental College, Lahore

Email address: saadiazahur.@live.com 